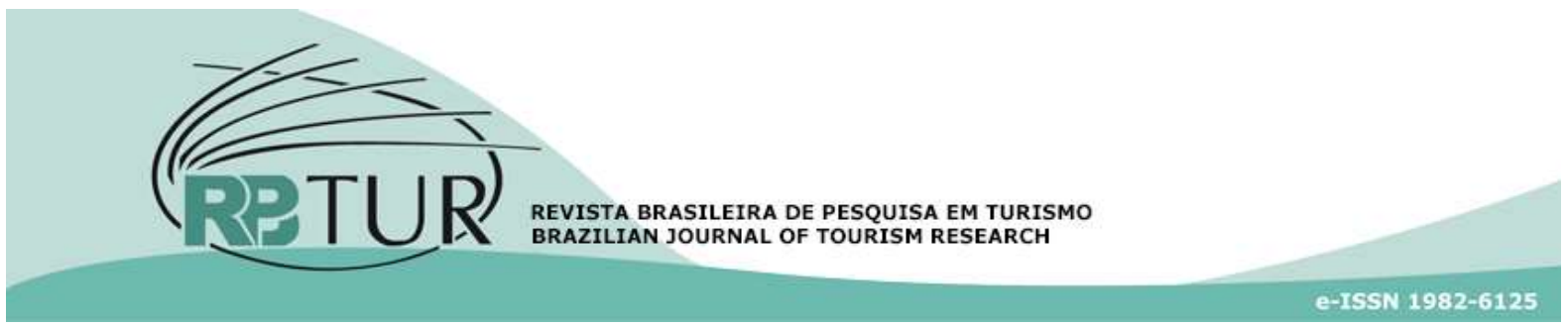

\title{
Autenticidade em Experiências de Turismo: proposição de um novo olhar baseado na Teoria da Complexidade de Edgar Morin*
}

\section{Authenticity in Tourism Experiences: a new approach based on Edgar Morin's Complexity Theory}

\section{Autenticidad en Experiencias de Turismo: propuesta de una nueva mirada basada en la Teoría de la Complejidad de Edgar Morin}

\author{
Mariana Bueno de Andrade Matos ${ }^{1}$ \\ Maria de Lourdes de Azevedo Barbosa ${ }^{2}$
}

Resumo: Este ensaio tem o objetivo de fornecer uma lente alternativa para interpretação do construto autenticidade no contexto das experiências turísticas. A revisão de literatura realizada apresenta os principais autores que tratam do tema e suas diferentes perspectivas a respeito de autenticidade, dentre estas a autenticidade objetiva, a construtivista e a existencial. A análise e reflexão dessas diferentes perspectivas oferecem as bases para a proposição da adoção de um solo epistemológico alternativo à interpretação da autenticidade nas experiências turísticas, que pode levar a uma melhor compreensão desse fenômeno. Assim, propõe-se que o Pensamento Complexo de Edgar Morin pode oferecer uma forma complementar para o entendimento da autenticidade no contexto do turismo, na medida em que pode permite ao pesquisador uma alternativa frente ao pensamento fragmentado tradicional que está atrelado a essa temática, e possibilitar reflexões a partir de uma visão mais holística do fenômeno. Essa discussão resultou em proposições, que são discutidas ao final deste trabalho.

Palavras-chave: Autenticidade. Turismo. Pensamento Complexo. Edgar Morin.

Abstract: This essay aims to provide an alternative lens for interpreting the construct 'authenticity' in the context of tourist experiences. The literature review presents the main authors of the field and its different known types

${ }^{1}$ Universidade Federal da Paraíba (DTH/UFPB), Joao Pessoa, PB, Brasil.

${ }^{2}$ Universidade Federal de Pernambuco (DHT/UFPE), Recife, PB, Brasil.

* Este estudo foi elaborado durante o doutoramento da primeira autora, sob orientação da segunda autora, e contou com financiamento do Conselho Nacional de Desenvolvimento Científico e Tecnológico (CNPq).

Artigo recebido em: 15/05/2018. Artigo aceito em: 08/08/2018.

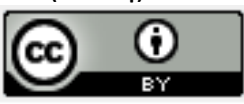


of authenticity, among them the objective, constructivist and existential authenticity. The analysis and reflection of these different perspectives provide the basis for proposing the adoption of an alternative epistemological ground to the interpretation of authenticity in tourist experiences, which may lead to a better understanding of this phenomenon. Thus, it is proposed that Edgar Morin's Complexity Theory may offer a complementary view to the understanding of authenticity in the context of tourism, insofar as it may allow an alternative to the traditional fragmented way of thinking, and reflections from a more holistic view of the phenomenon. This discussion resulted in propositions, which are discussed at the end of this paper.

Keywords: Authenticity. Tourism. Complexity Thinking Theory. Edgar Morin.

Resumen: Este ensayo tiene el objetivo de proporcionar una lente alternativa para la interpretación del constructo autenticidad en el contexto de las experiencias turísticas. La revisión de literatura realizada presenta los principales autores que tratan del tema y sus diferentes perspectivas respecto de autenticidad, entre ellas la autenticidad objetiva, la constructivista y la existencial. El análisis y reflexión de estas diferentes perspectivas ofrecen las bases para la proposición de la adopción de un suelo epistemológico alternativo a la interpretación de la autenticidad en las experiencias turísticas, que puede llevar a una mejor comprensión de ese fenómeno. Así, se propone que el Pensamiento Complejo de Edgar Morin puede ofrecer una forma complementaria para el entendimiento de la autenticidad en el contexto del turismo, en la medida en que puede permitir al investigador una alternativa frente al pensamiento fragmentado tradicional que está vinculado a esa temática, y que permite reflexiones a partir de una visión más holística del fenómeno. Esta discusión resultó en proposiciones, que se discuten al final de este trabajo.

Palabras clave: Autenticidad. Turismo. Teoría de la Complejidad. Edgar Morin.

\section{INTRODUÇÃO}

A origem dos estudos de autenticidade se deu quando debates sobre o que é real e o que não é real no contexto das atividades turísticas vieram à tona em função do processo de globalização, no início dos anos 1960. O pioneiro foi Boostin (2011), em seu livro The Image, publicado em 1962. Em sua obra não é citada a palavra 'autenticidade', mas a discussão sobre o tema percorre todo o seu livro, sendo ele considerado o primeiro autor a discutir o assunto (Maccannel, 1973; Cohen, 1979; Urry, 1990; Chambers, 2000). Boorstin (1962), analisou as experiências de turismo nos Estados Unidos e fez, em seu estudo seminal, uma crítica à sociedade do espetáculo. Para o autor, as pessoas estavam em busca permanente de eventos que as distanciasse da realidade do cotidiano. Além disso, o controle da natureza e o padrão de vida cada vez mais alto da população seriam as principais razões para que as pessoas criassem expectativas muito altas em relação às experiências turísticas, as quais só poderiam ser atingidas por meio do que ele chamou de 'pseudo-eventos', que seriam experiências, produtos e eventos fabricados para surpreender os turistas, afastando-os da realidade e, portanto, da autenticidade do produto turístico.

Uma outra linha de pensamento sobre a autenticidade é iniciada pelo também sociólogo MacCannel (1973), no início da década de 1970. O autor corrobora com Boorstin (1962) no que diz respeito à inautenticidade e o modo superficial da vida social à época, mas a principal diferença nas visões dos dois é que, ao contrário de seu antecessor, MacCannel (1973) defende que os turistas sempre viajam para buscar experiências autênticas e verdadeiras, e não apenas para repetir padrões e ficarem em suas zonas de conforto. O turismo seria uma nova forma de 
religião, e os turistas seriam peregrinos em busca de conhecer o mundo e suas culturas autênticas. Ele propôs, porém, baseado nos conceitos de Goffman (1956) de frontstage e backstage, que existem dois tipos de autenticidade: a real e a encenada - ou, em inglês, staged authenticity. A autenticidade real é o existente, e a encenada é a que é 'decorada' para ser percebida pelo turista como a real, que é, por sua vez, aquela que irá caracterizar o lugar na sua perspectiva de visitante temporário.

Assim como no princípio, em que Boorstin (1962) e McCannel (1973) não encontram um consenso sobre a autenticidade nas experiências turísticas, ao longo dos estudos a respeito do tema as discordâncias e dúvidas persistiram.

Ning Wang (1999), sociólogo da Universidade Zhongshan, China, que estuda principalmente sociologia do turismo, propôs uma organização e padrões para os conceitos de autenticidade existentes nos estudos em sociologia do turismo.

Em busca de novos conhecimentos e explicações para autenticidade, autores propõem que apenas alguns dos tipos de autenticidade conhecidos são válidos ou buscam combiná-los entre si (Reisinger; Steiner, 2006; Kim; Jamal, 2007; Belhassem, Caton, Stewart, 2008; Lau, 2010; Chhabra, 2010). No entanto, esta combinação pode ser questionada por conta de inconsistências epistemológicas com os paradigmas sobre os quais os conceitos estão apoiados, como foi destacado por Steiner e Reisinger (2006). Como exemplo, pode-se citar que a autenticidade objetiva está baseada no positivismo enquanto a autenticidade existencial baseia-se no existencialismo. Desse modo, a combina- ção das autenticidades torna-se algo não científico se feito com base nos paradigmas clássicos do conhecimento.

Diante da falta de consenso das bases teóricas que sustentam os estudos da autenticidade, busca-se com este ensaio contribuir com a discussão do tema e propor o que entende-se ser uma reflexão inovadora que pode auxiliar no debate sobre a autenticidade nas experiências turísticas, propondose como objetivo a análise da autenticidade a partir da Teoria da Complexidade do filósofo francês Edgar Morin (2010), que defende que para que se consiga compreender melhor o mundo moderno deve-se abandonar o pensamento e os paradigmas simplificadores e aderir a uma forma mais holística de visão de mundo, que seja capaz de compreender a complexidade dos fenômenos tal como ocorrem.

\section{A ORIGEM DOS CONCEITOS DE AUTENTI- CIDADE EM EXPERIÊNCIAS TURÍSTICAS}

A autenticidade em experiências de turismo é, segundo Cohen (1979b), um dos assuntos principais a serem discutidos quando estuda-se sociologia do turismo. No entanto, é possível encontrar estudiosos de antropologia (Bruner, 1994; Robinson, 1997; Santana, 2009), geografia (Delignières, 2015; Waitt, 2000; Rickly-Boyd, 2013), e arquitetura (Jokilehto, 2006; Stovel, 2007) que também estudam o fenômeno. No que diz respeito à autenticidade como construto independente ao turismo, ela é estudada ainda por administradores, que defendem a autenticidade como instrumento de liderança (Gilmore, 2007), linguistas (Macdonald; Badger; Dasli, 2006; Pietik; Kelly-Holmes, 2011), his- 
toriadores (Richman, 2008), engenheiros de alimentos e químicos.

As definições para o termo, como pode-se prever, variam de acordo com a abordagem e área científica em que está sendo estudada a autenticidade. Taylor (2001) afirma que existem tantas definições de autenticidade quanto pessoas que escrevem sobre o tema. De modo geral, o significado de autenticidade é "o caráter do que é genuíno, verdadeiro" (Houaiss, 2004, p. 77). No contexto das experiências de turismo também há discussão quanto ao conceito do termo e Cohen (1988) afirma que isso é um problema nas pesquisas sobre autenticidade. E esse autor complementa que a maioria dos turistas que são pesquisados sequer têm propriedade do significado do que estão sendo questionados, o que enfraquece as pesquisas. Bruner (1994) corrobora com o autor supracitado e afirma que a autenticidade é uma questão que está muito mais presente na mente dos pesquisadores ocidentais do que na mente dos turistas e nativos das regiões de turismo. Existem ainda as discordâncias a respeito dos conceitos entre os próprios pesquisadores, outro fato que dificulta a evolução dos estudos em autenticidade.

Diante desses problemas de discordância e demasiados conceitos para o mesmo fenômeno, Ning Wang (1999) organizou e estabeleceu padrões nos conceitos previamente discutidos por outros autores dos conceitos existentes de autenticidade e desenvolveu e amadureceu o conceito de autenticidade existencial, o que contribuiu para a evolução do conhecimento na área.

Wang (1999) apresenta diversos conceitos pregressos adotados e, ao fim, estrutura de maneira sistematizada as definições.
Ele afirma que existem três tipos de autenticidade: a objetiva, a construtivista e a existencial (da pós-modernidade). A autenticidade objetiva utiliza-se de conceitos que têm origem nos estudos de museologia, ou seja, nas características físicas, à originalidade de artefatos, por exemplo. Existem publicações que visam analisar questões da autenticidade objetiva em turismo: pesquisas sobre souvenires (Littrel; Anderson; Brown, 1993); pesquisas que buscam compreender a relação do turista com a localidade por meio de análises objetivas e físicas (Wheitman, 1987), dentre outras. Os estudiosos de geografia física, particularmente, utilizam-se bastante desse tipo de autenticidade para analisar as relações do turista com o local (Baudrillard, 1986; Waitt, 2000).

A autenticidade construtivista é, por sua vez, fruto dos estudos e dos autores que afirmam que a percepção de autêntico é uma construção social e é algo negociável. Ela acontece quando há consenso social, por meio de um grupo de pessoas, por exemplo, de que aquele fato, experiência ou fenômeno são autênticos (Moscardo; Pearce, 1986; Cohen, 1988; Ehrentraut, 1993; Bruner, 1994).

Por fim, a autenticidade existencial é, para Wang (1999), aquela que leva em conta questões pessoais de cada turista/indivíduo, tais como seus sentimentos e percepções para analisar a experiência. Antes de Wang (1999) autores deram suas contribuições para conseguir chegar ao termo 'autenticidade existencial'. Cohen (1979b) contribuiu com a afirmação de que cada turista têm uma experiência diferente e que essas particularidades influenciam a percepção de autenticidade, e Gottlieb (1982) despertou reflexões a respeito da individualidade de cada turista. 
Este autor complementa que a origem do turista e suas características particulares (classe social, estilo de vida) influenciam no que cada turista busca e como ele se sente. Para Gotlieb (1982), por exemplo, os turistas que são ricos em sua residência têm maior inclinação a buscar experiências simples, já os turistas que são de classes sociais inferiores buscariam mais experiências de luxo, tendo em vista que todos buscam o que lhe é diferente e exótico. Esse fenômeno ele chama de "rainha/rei por um dia e/ou mendigo por um dia". Sobre essa transição de classe social temporária, Harkin (1995) também trata, mas apenas do ponto de vista da transição para uma classe social superior, ele afirma que o turista pode em uma viagem ocupar uma posição que não pode sustentar em seu dia-a-dia. Assim, o turismo no terceiro mundo expressa, segundo o autor, uma nostalgia do colonialismo (Harkin, 1995, p. 652).

Após o início da reflexão do papel de cada indivíduo e suas particularidades sobre a percepção e construção da autenticidade, vários estudos surgiram levando esses fatos em conta e atendo-se mais ao humano na atividade de turismo, tais como: a percepção de autenticidade teria a ver com a satisfação (Moscardo; Pearce, 1986); crítica ao turismo de massa por isolar os turistas do autêntico (Weightman, 1987); os riscos da comoditização do turismo para a autenticidade (Cohen, 1988); divisão dos turistas em tipos a partir de sua relação com a autenticidade (Silver, 1993). Por fim, George Hughes (1995), geógrafo da Universidade de Edimburgo, na Inglaterra, chegou ao termo 'existencial' ao romper com as ideias de que a autenticidade seria uma construção social.

$\mathrm{O}$ termo autenticidade existencial, se gundo alguns autores, vem do filósofo existencialista alemão Heidegger (Steiner; Reisinger, 2006; Grunewald, 2007) e não surgiu com os estudos de turismo, e sim muito antes com as ideias iniciais de filósofos como Sartre, Rousseau e Kant (Steiner; Reisinger, 2006). No entanto, as aplicações do conceito em turismo surgiram de acordo com o que foi explicitado anteriormente a este parágrafo.

É importante destacar que originalmente as três tipologias de Autenticidade foram sendo pensadas como uma evolução, os autores construtivistas discordam dos objetivistas, e ambos são, por sua vez, criticados pelos existencialistas.

\section{A ATUALIDADE DOS ESTUDOS EM AUTEN- TICIDADE}

Após a organização dos conceitos realizada por Wang (1999), as discussões passaram a ser mais subdivididas entre os três tipos de autenticidade (Reisinger; Steiner, 2006; Kim; Jamal, 2007; Lau, 2010) e os autores passaram a se posicionar e defender uma ou outra abordagem e tipo de autenticidade. Apesar do grande interesse pelo tema, alguns estudiosos continuaram defendendo a falta de relevância dos estudos sobre autenticidade (Taylor, 2001) por conta das grandes variáveis que envolvem a percepção de que algo é ou não autêntico. É como discutir sobre o que é a verdade, que entra em esferas filosóficas e ontológicas bastante individuais. Por outro lado existem pesquisas que comprovam que existem turistas que estão interessados na autenticidade de experiências, ou seja, buscam vivências originais, aproximar-se de manifestações culturais ainda não exploradas turisticamente, e buscam o pito- 
resco, o local. Desse modo, turistas demonstram preocupar-se com a autenticidade da localidade que visitam (Mkono, 2012). Acredita-se que juntamente com a globalização e o excesso de homogeneização dos produtos e serviços oferecidos que vêm à tona com a modernidade contemporânea, surge uma força de resistência por meio de uma maior valorização local (Santos, 2011). Essa força de resistência desperta nas pessoas a busca por experiências e vivências mais autênticas e, por isso, na contramão desse turbilhão que é a globalização, surgem opções de consumo alternativo que possuem público cada vez maior, e as experiências autênticas estão neste caminho. Exemplos disso são iniciativas de consumo colaborativo, inclusive em turismo, tais como os programas Dineer, Rent a Local Friend, Couch Surfing, dentre outros. Portanto, o turismo de massa torna-se cada vez mais forte, sim, mas na contramão a isso existe um outro tipo de turista que procura a autenticidade em suas viagens (Belhassem; Caton, 2006; Mkono, 2012). Desse modo, especialmente para o turismo cultural, étnico e histórico, a autenticidade é fundamental (Wang, 1999).

Em busca de novos conhecimentos e explicações para autenticidade, autores propõem que apenas alguns dos tipos de autenticidade conhecidos são válidos (Reisinger; Steiner, 2006; Kim; Jamal, 2007; Lau, 2010). Reisinger e Steiner (2006) propõem o abandono da autenticidade objetiva e Lau (2010) afirma que apenas a objetiva existe, por exemplo. Seguindo outros caminhos, autores buscam agrupar alguns tipos de autenticidade em suas análises para melhor compreendê-la como um todo. Este é o caso de BeIhassem, Caton e Stewart (2008), os quais montam um modelo teórico que busca meIhor compreender as relações com a autenticidade dos turistas peregrinos religiosos na Terra Santa. Os autores chegam ao conceito de autenticidade 'THEOPLACITY', que seria a união das crenças dos turistas, com o lugar e as atividades. Esses seriam os fatores para que a vivência seja autêntica no contexto estudado, e eles defendem a união do que seria a autenticidade objetiva (lugar) e a autenticidade existencial (atividades).

Chhabra (2010), por sua vez, propõe o conceito de autenticidade 'Negociada' (negotiated), o qual afirma ser o primeiro conceito que leva em conta a segmentação de mercado para analisar autenticidade. Ele analisa turistas por idade (geração) e estilo de vida, tentando compreender a relação da geração $Y$ com a autenticidade. Em seu estudo ele sugere que a autenticidade negociada é um trade-off entre a autenticidade objetiva e a autenticidade construtivista.

Essa união de tipos de autenticidade, no entanto, pode ser contestada por conta de problemas epistemológicos com os paradigmas e modos de pensar aos quais estão baseados (Steiner; Reisinger, 2006). A autenticidade existencial, por exemplo, baseia-se no existencialismo, enquanto a construtivista, no construtivismo, que são visões de mundo diferentes entre si.

A pesquisadora Rickly-Bold (2012) baseia-se na teoria de Walter Benjamim, sociólogo da escola de Frankfurt, para defender que a autenticidade é um construto que deve ser estudado de maneira global. Ela defende que objeto, lugar e experiência devem ser unidos em um framework de autenticidade. A partir disso, a autora busca na teoria de Benjamin, a respeito de aura e autenticidade, 
respaldo para seus argumentos. A aura, a princípio, é propriedade sentida por pessoas a partir do contato com um objeto, cidade ou lugar. E esse sentimento é capaz de ser sentido mesmo depois que o momento de contato acontece, como é o caso do que ocorre com os souvenirs e fotografias. A partir disso, a autora afirma que a autenticidade é relacional, ela acontece a partir da relação entre duas coisas, especialmente tradições, rituais e aura. Afirma ainda que a autenticidade pode ser ao mesmo tempo medida, sentida e experienciada.

O estudo de Rickly-Boyd (2012) contribui principalmente como retorno à preocupação com o objeto, tão necessária, já que o turismo depende do ambiente físico para acontecer, e não apenas de alguém sentindo uma sensação de autenticidade a partir de uma ação individual. No entanto, acredita-se que pode-se ir além. Na próxima seção é apresentada a nossa proposta.

\section{RUMO À COMPLEXIDADE DA AUTENTICI DADE NAS EXPERIÊNCIAS TURÍSTICAS}

Assim como ficou demonstrado na seção anterior a tendência dos últimos autores é a de combinar 'tipos' de autenticidade debruçando-se em mais de uma característica da experiência (Belhassem; Caton; Stewart, 2008; Chhabra, 2010; Rickly-Boyd, 2012). O que se propõe neste ensaio é avançar um pouco mais nas reflexões sobre autenticidade e estudá-la de forma mais contextualizada, propondo que o objeto, o meio social, as questões individuais dos turistas e todo o ambiente em que a experiência de turismo acontece devem ser interpretados de forma holística, como ocorre na prática, e não de maneira simplificada e fragmentada como veio ocorrendo desde o início dos estudos sobre autenticidade.

É importante esclarecer que este $p a-$ per não tem a intensão de propor novos conceitos de autenticidade para aumentar ainda mais a lista de subtipos e variações existentes e tornar o debate sobre o tema ainda mais complexo do que já denuncia Taylor (2001). O que se pretende é propor um avanço no debate, a partir da busca da compreensão da autenticidade em experiências de turismo adotando uma forma de pesquisa que seja capaz de unir as teorias já estabelecidas, mas também permitir reflexões diferentes. Dessa forma, acredita-se que se poderá oferecer aos pesquisadores um meio de aproximação da realidade do fenômeno para melhor compreender as vivências dos turistas.

No sentido de uma proposição alternativa ao pensamento vigente a respeito da autenticidade, levou-se em consideração reflexões de autores que vem debatendo o tema, como Mkono (2012), que considera que os estudos sobre autenticidade devem ser mais situacionais para que os conceitos deixem de ser tão eurocêntricos. Do mesmo modo, Grunewald (2004) afirma que critérios fixos são incapazes de analisar autenticidade, já que este acontece em um meio social específico. Dessa forma, propõe-se a introdução de um modo de pensar que seja capaz de, sem estabelecer parâmetros fixos, analisar questões particulares de acordo com o contexto de cada experiência de turismo.

Assim, este estudo sugere o olhar sobre a autenticidade baseando-se na Teoria do Pensamento Complexo. Esta forma de pensar permite combinar os elementos das teorias existentes e fazer emergir questões 
novas, a partir da análise contextualizada do fenômeno. A Complexidade foi desenvolvida pelo filósofo francês Edgar Morin (2011) que afirma que para se entender e passar a compreender melhor o mundo moderno, deve-se abandonar o pensamento fragmentado e hipersimplificado, pois este reduz o mundo à pequenas partes que são incapazes de apresentar respostas coerentes.

A partir disso, Morin (2011) afirma que a complexidade não vê o homem como um ruído (como no positivismo), tampouco ignora o objeto e olha apenas para o homem desprovido de um contexto (como no humanismo). Considera, no entanto, a interdependência sistema/ecossistema, e afirma que "o mundo está no interior de nossa mente, que está no interior do mundo" (2011, p. 43).

Assim, a teoria da complexidade baseia-se nos sistemas abertos e o autor defende que o homem precisa compreender que sujeitos e objetos que são considerados absolutos deixam de ver uma fenda enorme de conhecimento entre eles. Mas, a partir do momento em que se reconhece esse 'abismo' eles passam a estar abertos para novas possibilidades que podem levar a novos conhecimentos, realizando, assim, um progresso na ciência.

É possível traçar um paralelo dessas reflexões de Morin (2011) com a teoria existente sobre autenticidade. A partir do momento em que se abandona a supremacia da 'autenticidade existencial' (do homem) ou a superioridade da 'autenticidade objetiva', é possivel reconhecer que entre esses dois elementos da experiência turística, homem e objeto, existe uma grande quantidade de possibilidades de análise e evolução do conhecimento na área, como já defendem al guns autores de autenticidade que buscamcombinar os conceitos em experiências turísticas (Grunewald, 2007; Belhassem; Caton, 2006; Chhabra, 2010; Mkono, 2012).

Para tanto, é relevante esclarecer que o Pensamento Complexo de Morin (2011) não pretende ser o único paradigma possível, tampouco superior aos demais conhecimentos desenvolvidos, ele pode sim ser capaz de agregar aos conhecimentos anteriores, sem as suas limitações e cegueiras, oferecendo a possibilidade de observar o todo com perspectivas mais amplas e coerentes à imensidão de fatores nos quais as realidades estão inseridas. Nas palavras do autor:

A scienza nuova não destrói as alternativas clássicas, não oferece solução monista como se fosse a essência da verdade. Mas os termos alternativos tornam-se antagônicos, contraditórios, e ao mesmo tempo complementares no seio de uma visão mais ampla, que vai precisar reencontrar e se confrontar com novas alternativas. (Morin, 2011, p. 53)

Se faz necessário esclarecer que no caminho seguido por Morin (2011) as teorias clássicas perdem o seu caráter absoluto, deixam de ser reducionistas. No entanto, é importante destacar que o que se busca também não é o holismo, que pode querer agregar a totalidade, mas que quanto mais plena torna-se mais vazia. O que se busca é a unidade complexa, é unir o pensamento analítico/reducionista à globalidade, numa dialetização que seja capaz de compreender que a parte está no todo e que o todo só existe por conta da parte.

Sendo assim, este estudo propõe a adoção do paradigma da complexidade como meio capaz de compreender a autenticidade em experiências de turismo. $O$ que se busca 
é livrar-se das amarras que fragmentam as filosofias previamente utilizadas para o desenvolvimento do conhecimento em autenticidade das experiências turísticas (existencialismo, positivismo e construtivismo), para poder unir as visões desenvolvidas, mas tendo consciência de que são partes integrantes de um todo complexo. Como visto, a teoria de Morin tem a intenção de ser um sistema aberto e que permita a convivência e a coexistência de diferentes formas de pensar, e que todas são partes do todo. Esse todo é a complexidade. Tais afirmações podem ser observadas na citação:

A complexidade surge, é verdade, lá onde o pensamento simplificador falha, mas ela integra tudo o que põe ordem, clareza, distinção, precisão do conhecimento. Enquanto o pensamento simplificador desintegra a complexidade do real, o pensamento complexo integra o mais possível os modos simplificadores de pensar, mas recusa as consequências mutiladoras, redutoras, unidimensionais e finalmente ofuscantes de uma simplificação que considera reflexo do que há de real na realidade (Morin, 2011, p.6)

Assim, acredita-se e defende-se que apenas com a adoção de um novo olhar, a partir de um solo epistemológico alternativo, é que uma reflexão mais profunda será capaz de compreender e explicar algumas questões ainda não respondidas.

O paradigma permite compreender o fenômeno de maneira singular por observálo considerando a maior complexidade possível, e não apenas limitando-se a uma esfera ou ator (objeto ou meio social ou consumidor). O paradigma da complexidade contribui, a partir do momento em que analisa as diversas possibilidades e visões. Cohen (1979b) já defendia em sua teoria que todas as partes envolvidas (atores) são importantes para a autenticidade das experiências turísticas.

Assim, a filosofia de Morin (2011) permite não só que o ponto de vista dos turistas, mas também da população local, dos prestadores de serviços e demais envolvidos, bem como os aspectos físicos sejam observados e, assim, o conjunto seja melhor estudado.

Além dos atores, o meio social também é fundamental, já que a experiência de um turista influencia e é influenciada pela experiência dos demais, segundo a teoria de marketing experiencial (Petr, 2002). Do mesmo modo, Mclntosh e Prentice (1999), autores de autenticidade, destacam que as relações pessoais e as emoções durante a vivência influenciam a percepção de autenticidade, inclusive a autenticidade física do lugar.

Propõe-se, portanto, que não apenas a autenticidade dos artefatos, atrativos físicos e objetos, tampouco o meio social isolado, nem as ações e características dos indivíduos (sejam a população local ou os turistas) isoladamente são importantes, todas precisam umas das outras para existir e formar o 'todo' da experiência. O pensamento também permite que as proposições a serem feitas sejam utilizadas para compreender a vivência, enquanto pesquisador (que tem suas particularidades por conhecer conceitos e teorias a respeito do tema), bem como para analisar as experiências de outrem e para perceber as nuances entre diferentes experiências em locais diferentes. Assim, propõe-se que o estudo da autenticidade possa englobar concepção (ou não concepção), compreensão e experiência da autenticidade. 
Além dos conceitos e teorias já estudados por autores anteriores, propõe-se que alguns fatores de análise devem ser tratados no cenário das experiências turísticas, já que podem influenciar as percepções e experiências dos indivíduos: as iniciativas governamentais, os esforços de marketing, a forma como se constrói a marca do destino turístico (que gera expectativas que influenciam a experiência) e questões situacionais e particulares de cada experiência.

As iniciativas governamentais e todas as questões próprias de cada situação são relevantes para compreender o todo. Os esforços de marketing e o governo já haviam sido citados por Steiner e Reisinger (2006) como relevantes na atração dos turistas. Acreditase que são também importantes para a percepção de autenticidade pelo turista, e por meio da teoria de Marca de Destinos Turísticos é possível afirmar que a imagem que o turista tem antes da viagem influencia a sua experiência já que gera algum tipo de expectativa (positiva ou negativa) nesta. Do mesmo modo, os esforços promocionais e a identidade dos destinos (como a população e o governo enxergam o destino) também são importantes na percepção e na vivência da experiência turística (Molanen; Rainisto, 2009). Bruner (1991), antropólogo que possui pesquisas em autenticidade, já na década de 1990, afirmava que muitas vezes as promessas de serviço poderiam ser muito diferentes das experiências de turismo, e Pocock (1992) descreve em seu trabalho a influência que um livro, que romantiza uma localidade, pode influenciar e distorcer a percepção que um turista possui de um local. Chhabra, Hearly e Sills (2003) também tratam da importância do marketing em seus estudos. Eles re- latam que a percepção de autenticidade é controlada pela mídia e pelo boca-a-boca.

Além dessas questões de promoção, marketing e do governo, que é quem promove, financia e apoia muitas atividades ligadas ao turismo, outras questões situacionais da localidade (ter maior ou menos relação com governo, estar isolada geograficamente, dentre outros) ou particulares da vivência de cada turista (que pode sofrer um acidente, ou ter relação especial com a localidade, pode ainda perder um vôo, uma reserva, dentre outros) podem emergir (Grunewald, 2004; Mkono, 2010) durante o processo de experiência ou compreensão de uma vivência. Assim, e de acordo com a teoria de Morin (2011), propõe-se um sistema de interação aberto entre as características e situações citadas. Portanto, por meio da maneira de pensar concebida por Morin (2011), propõe-se a busca pela compreensão do todo, mas também das partes que o formam, a fim de meIhor compreender a importância, as relações e a concepção da autenticidade nas experiências de turismo de maneira global.

\section{CONSIDERAÇÕES FINAIS}

Por meio do apresentado, é possível perceber que a partir do surgimento das primeiras discussões sobre autenticidade, na década de 1960, diversas linhas de pensamento surgiram com relação a este construto. Antropólogos, sociólogos, arquitetos, geógrafos e pesquisadores de ainda outras áreas estudam o fenômeno, sempre de acordo com seus objetos de pesquisa. Desse modo, e com maior ênfase em sociologia e turismo, foram apresentadas correntes de pensamento que deram origem a três princi- 
pais tipos de autenticidade: a objetiva, a construtivista e a existencial (da pós-modernidade).

A partir do fim da década de 1990, no entanto, autores vêm buscando combinar ou defender a supremacia de alguns aspectos em detrimentos de outros dentro do cenário de autenticidade. No entanto, este estudo propõe um diferente solo epistemológico a fim de que se consiga um novo olhar da autenticidade em experiências turísticas. Este solo é a Teoria do Pensamento Complexo de Edgar Morin.

Deste modo, as teorias clássicas utilizadas para embasar as discussões baseadas no positivismo, construtivismo e existencialismo perdem o seu caráter absoluto e o que se busca é a compreensão mais complexa das realidades estudadas. Assim, pode-se direcionar ao fenômeno um olhar mais cuidadoso de seus diferentes aspectos e realidades, a fim de que se consiga ter maior proximidade das experiências como são vivenciadas pelos turistas, população local, prestadores de serviços, e todos os envolvidos na realidade estudada.

Assim, envolvemos uma grande variedade e peculiaridades de todos os atores e elementos da experiência (físicos, sociais, ambientais, de histórias, de perspectivas, a partir dos cargos de cada prestador de serviço, dentre outros). Todos compõem o ambiente em que ocorrem as vivências, influenciam e são influenciados, uns pelos outros.

A Teoria da Complexidade e a desfragmentação do conhecimento fornecem, destarte, o solo epistemológico apropriado para o estudo de um fenômeno complexo como é o turismo e especialmente para o es- tudo aprofundado da autenticidade nesses cenários.

Deste modo, e com base nos argumentos desenvolvidos neste estudo, elaboramos algumas proposições que podem ser estudadas ou aprofundadas em estudos futuros, são elas:

1. O estudo da autenticidade é relevante à medida em que as novas formas de consumo e o novo comportamento que os turistas vêm desenvolvendo com os destinos turísticos demonstram, cada vez mais, a busca por experiências próximas ao que se entende por questões culturais originais, visita a locais menos turísticos e maior relação com locais e seu dia-a-dia. O que se exemplifica por meio das novas formas de consumo colaborativo, turismo criativo e surgimento de plataformas como o Couch Surfing, Rent a Local Friend, dentre outros.

2. Para que a compreensão da autenticidade seja coerente e relevante com o que ocorre nas experiências turísticas, não se pode isolar aspectos físicos, aspectos sociais, questões individuais dos consumidores, questões individuais dos prestadores de serviço e questões individuais da população local.

3. O marketing, nomeadamente os esforços de promoção de marca de destino turístico ou de produto turístico, influenciam a percepção de autenticidade em experiências turísticas. Isto ocorre à medida 
que a imagem que o consumidor constrói antes mesmo da vivência gera expectativas que influenciam suas percepções.

4. A autenticidade de uma experiência é construída ao longo de todo o contato do consumidor com a comunidade receptora, sendo negociada a todo momento ao longo da vivência.

Deste modo, entende-se que estas proposições são capazes de gerar reflexões capazes de direcionar pesquisas futuras e, por sua vez, foram elaboradas e refletidas com base no olhar que a Teoria da Complexidade nos permitiu ter do fenômeno estudado. Deste modo, este artigo cumpre o seu objetivo, de fornecer uma lente alternativa para interpretação do construto autenticidade no contexto das experiências turísticas, a partir do momento em que demonstra a possibilidade de análise do construto com base na Teoria da Complexidade de Edgar Morin.

\section{REFERÊNCIAS}

Arnold, E. (1991) Authenticity revisited: How real is real? English for Specific Purposes, 10(3), p. 237-244. DOI: https://doi.org/10.1016/08894906(91)90027-T

Barbosa, M. L. A.; Farias, S. A.; Kovacs, M. H. (2008) Entre a fome e a vontade de comer: os significados da experiência de consumo em restaurantes. III encontro de marketing. Anais ... Rio de Janeiro: ANPAD.

Baudrillard, J. (1988) América. Paris: Bernard Grasset

Belhassen, Y. Caton, K. (2006) Authenticity Matters. Annals of Tourism Research, 33(3), p. 853-856.DOI: https://doi.org/10.1016/i.annals.2006.03.009
Belhassen, Y.; Caton, K.; Stewart, W. P. (2008) The search for authenticity in the pilgrim experience. Annals of Tourism Research, 35(3), p. 668-689. DOI: https://doi.org/10.1016/i.annals.2008.03.007

Boorstin, D. J. (1962) The image: a guide to pseudo-events in America. New York: Vintage Books.

Brown, L. (2013) Tourism: A catalyst for existential authenticity. Annals of Tourism Research, 40(1), p. 176-190. DOI: https://doi.org/10.1016/j.annals.2012.08.004

Bruner, E. M. (1991) Transformation of self in tourism. Annals of Tourism Research, 18(2), p. 238-250. DOI: https://doi.org/10.1016/01607383(91)90007-X

(1994) Abraham Lincoln as authentic reproduction: a critique of postmodernism. American Anthropologist, 96(2), p. 397-415. DOI: https://doi.org/10.1525/aa.1994.96.2.02a00070

(2009) The Study of Tourism: Anthropological and Sociological Beginnings. Annals of Tourism Research, 36(3), p. 547-549. DOI: https://doi.org/10.1016/i.annals.2008.07.006

Burrell, G.; Morgan, G. (1982) Sociological Paradigms and Organisational Analysis. Londres: Heinemann.

Carù A.; Cova B. (2006) Expériences de consommationet marketing expérientiel. Revue Française de Gestion, 32(162). DOI: https://doi.org/10.1177/14705931030032004

(2003) Revisiting Consumption Experience: a more humble but complete view of the concept. Marketing Theory, 3(2), p. 267-286.

Chambers, E. (2000) Native tours: the anthropology of travel and tourism. Long Grove: Waveland Press.

Chen, G. ( 2015) The tourist semiotic practice: Is the marker authentic? Annals of Tourism Research, 53, p. 101-104. DOI: https://doi.org/10.1016/j.annals.2015.04.006 
Chhabra, D. (2008) Positioning museums on an authenticity continuum. Annals of Tourism Research, 35(2), p. 427-447.

. (2012) Authenticity of the Objectively Authentic. Annals of Tourism Research, 39(1), p. 499-502.

Chhabra, D.; Healy, R.; Sills, E. (2003) Staged authenticity and heritage tourism. Annals of Tourism Research, 30(3), p. 702-719.DOI: https://doi.org/10.1016/j.annals.2007.12.001

Chronis, A ; Arnould, E. J; Hampton, R. D. (2012) Gettysburg re-imagined: the role of narrative imagination in consumption experience. Consumption Markets \& Culture, 15(3), 261-286. DOI:

https://doi.org/10.1080/10253866.2011.652823

Cohen, E. (1974) Who is a tourist?: a conceptual clarification.

.(1979a) A Phenomenology of Tourist Experiences. Sociology (sage), v. 1, p. 1-7.

(1979b) Rethinking the Sociology. Annals of Tourism Research, p. 18-35.

(1984) The sociology of tourism: approaches, issues and findings. Annual Review of Sociology, 10(1984), p. 373-392. DOI: https://doi.org/10.1146/annurev.so.10.080184.0021 $\underline{05}$

.(1988) Authenticity and Commoditization in Tourism. Annals of Tourism Research, 15, p. 371-386. DOI: https://doi.org/10.1016/01607383(88)90028-X

(1989) Primitive and remote: Hill tribe trekking in Thailand. Annals of Tourism Research, 16(1), p. 30-61. DOI: https://doi.org/10.1016/0160$\underline{7383(89) 90029-7}$

Cohen, E.; Avieli, N. (2004) Food in tourism Attraction and impediment. Annals of Tourism Research, 31(4), p. 755-778. DOI: https://doi.org/10.1016/j.annals.2004.02.003

Cohen, E.; Cohen, S. A. (2012a) Authentication: Hot and cool. Annals of Tourism Research, 39(3), p.

1295-1314.

DOI:

https://doi.org/10.1016/j.annals.2012.03.004

(2012b) Current sociological theories and issues in tourism. Annals of Tourism Research, 39(4), p. 2177-2202. DOI: https://doi.org/10.1016/i.annals.2012.07.009

Cole, S. (2007) Beyond authenticity and commodification. Annals of Tourism Research, 34(4), p. 943-960. DOI: https://doi.org/10.1016/i.annals.2007.05.004

Crang, M. (1996) Living history: magic kingdoms or a quixotic quest for authenticity ? Annals of Tourism Research, 23(2), p. 415-431. DOI: https://doi.org/10.1016/0160-7383(95)00070-4

Creswell, J. (2010) Projeto de Pesquisa: métodos qualitativo, quantitativo e misto. 3 ed. Porto Alegre: Artmed.

Csikszentmihalyi, M. (2000) Beyond Boredom and Anxiety. San Francisco: Jossey-Bass Publishers.

Daniel, Y. P. (1996) Tourism Dance Performances Authenticity and Creativity. Annals of Tourism Research, 23(4), p. 780-797. DOI: https://doi.org/10.1016/0160-7383(96)00020-5

Dann, G. M. S.; Parrinello, G. L. (2009) The sociology of tourism: European origins and developments. Tourism social science series, 39(1), p. 466

Delignières, V. (2015) L' authenticité des territoiresrurauxtouristiques en question: Réflexions au sujet de I' Auxoiset du Morvan. Revue Géographique de l'Est, p. 103-116.

Denzin, N.; Lincoln, Y. (1994) The Handbook of Qualitative Research.London: Sage Publications.

Di Betta, P. (2014) Authenticity as a mental state: A primer. Annals of Tourism Research, 47, p. 8688.

DOI:

https://doi.org/10.1016/j.annals.2014.05.002

Eco, U. (1986) Travels in Hyper-Reality. Orlando: Harcourt Brace \& Company. 
Ehrentraut, A. (1993) Heritage authenticity and domestic tourism in japan. Annals of Tourism Research, v. 20, p. 262-278. DOI: https://doi.org/10.1016/0160-7383(93)90054-7

Evans-Pritchard, D. (1989) How "they" see "us": Native American images of tourists. Annals of Tourism Research, 16(1), p. 89-105. DOI: https://doi.org/10.1016/0160-7383(89)90032-7

Fortuna, C. (1995) Turismo, Autenticidade e Cultura Urbana. Revista Crítica de Ciências Sociais.

Flick, U.; Kardorff, E.; Steike, I. (2004) A Companion to Qualitativa Research. London: Sage Publications.

Frochot, I.; Batat, W. (2013) Authenticity and commodification. Marketing and Designing the Tourist Experince, 28(3), p. 565-580.

Gnoth, J.; Wang, N. (2015) Authentic knowledge and empathy in tourism. Annals of Tourism Research, 50, p. 159-172. DOI: https://doi.org/10.1016/i.annals.2014.11.010

Gottlieb, A. (1982) Americans ' Vacation. Annals of Tourism Research, 9, p. 165-187. DOI: https://doi.org/10.1016/0160-7383(82)90045-7

Grabum, N. H. (1983) The anthropology of tourism. Annals of Tourism Research, 10, p. 9-33. DOI: https://doi.org/10.1016/0160-7383(83)90113-5

Greenwood, D. J. (1976) Tourism as an agent of change: a Spanish Basque case. Annals of tourism research, 3(3), p. 128-142. DOI: https://doi.org/10.1016/0160-7383(76)90005-0

Grunewald, R. A. (2004) The contingency os authenticity: intercultural experiences in indigenous villages os eastern and northeastern Brazil. Vibrant, 6(2), p. 225-253.

Gupta, S; Vajic, M. (2000) The contextual and dialectical nature of experience, new service development, creating memorable experiences in: Fitzsimmons, J.A. \& Fitzsimmons, M.J. (ed.). Thousand Oaks: Sage.
Hall, C.M.; Sharples, L; Mitchel, R.; Macionis, N.; Cambourne, B. (2003) Food Tourism Around the World: Development, management and markets. Oxford: Butterworth Heinemann.

Hanlan, J.; Kelly, S. (2005) Image formation, information sources and an iconic Australian tourist destination. Journal of Vacation Marketing, 11(2), p. 163-177. DOI: https://doi.org/10.1177/1356766705052573

Harkin, M. (1995) Modernist Anthropology And Tourism Of The Authentic. Annals of Tourism Research, 22(3), p. 650-670. DOI: https://doi.org/10.1016/0160-7383(95)00008-T

Hirschman, E. C; Holbrook, M. B. (1982) Hedonic consumption: emerging concepts, methods and propositions. Journal of Marketing, 46, Summer, p. 92-101. DOI: https://doi.org/10.2307/1251707

Holbrook, M. B. (2006) The consumption Experience - Somethig new, something old, Something borrowed, something sold: part 1. Journal of Macromarketing, 26, p. 259. DOI: https://doi.org/10.1177/0276146706291064

. (2000) The Millennial Consumer in the

Texts of Our Times: Experience and Entertainment. Journal of Macromarketing, 20(2), 178-192. DOI: https://doi.org/10.1177/0276146700202008

Hoffman, K. D.; Bateson, J. E. G.; Ikeda, A. A.; Campomar, M. C. (2010) Princípios de Marketing de Serviços: conceitos, estratégias e casos. 3 ed. São Paulo: Cengage Learning.

Houaiss, A. (2004) Minidicionário Houaiss da Língua Portuguesa. 2.ed. Rio de Janeiro: Objetiva.

Hughes, G. (1995) Authenticity in Tourism. Annals of Tourism Research, 22(4), p. 781-803. DOI: https://doi.org/10.1016/0160-7383(95)00020-X

Jokilehto, J. (2006) Considerations on authenticity and integrity in World Heritage context. City \& Time, 2(1), p. 1-16.

Kim, H.; Jamal, T. (2007) Touristic quest for existential authenticity. Annals of Tourism 
Research, 34(1), p. 181-201. DOI: https://doi.org/10.1016/j.annals.2006.07.009

Kirillova, K.; Lehto, X. (2015) An existential conceptualization of the vacation cycle. Annals of Tourism Research, 55, p. 110-123. DOI: https://doi.org/10.1016/i.annals.2015.09.003

Knudsen, D. C.; Rickly, J. M.; Vidon, E. (2016) S. The fantasy of authenticity: Touring with Lacan. Annals of Tourism Research, 58, p. 33-45. DOI: https://doi.org/10.1016/i.annals.2016.02.003

Kolar, T.; Zabkar, V. (2010) A consumer-based model of authenticity: An oxymoron or the foundation of cultural heritage marketing? Tourism Management, 31(5), p. 652-664. DOI: https://doi.org/10.1016/j.tourman.2009.07.010

Kohler, A. (2009) Autenticidade: origens e bases da discussão em turismo. Turismo: visão e ação. $11(3)$, p. 282-303.

Kuhn, T. (1998) A estrutura das revoluções científicas. São Paulo: Perspectivas.

Lau, R. W. K. (2010) Revisiting authenticity: A social realist approach. Annals of Tourism Research, 37(2), p. 478-498. DOI: https://doi.org/10.1016/i.annals.2009.11.002

Lithe, M. A.; Anderson, L. F.; Brown, P. J. (1993) What Makes A Craft Souvenir Authentic? Annals of Tourism Research, 20, p. 197-215. DOI: https://doi.org/10.1016/0160-7383(93)90118-M

Llosa, M. V.( 2009) The Culture of Liberty. Foreign Policy, p. 66-71.

Maccannel, D. (1973) Staged Authenticity: arrangements of social space in tourism settings. The American Journal of Sociology, 79(3), p. 589603. DOI: https://doi.org/10.1086/225585

(2014) Comment on Lau and Knudsen/Rickly-Boyd. Annals of Tourism Research, 44(1), p. 283-287.

Macdonald, M. N.; Badger, R.; Dasli, M. (2006) Authenticity, Culture and Language Learning. Language and Intercultural Communication, 613-
4), p. 250-261. DOI: https://doi.org/10.2167/laic252.0

Martin, K. (2010) Living pasts: Contested tourism authenticities. Annals of Tourism Research, 37(2), p. 537-554. DOI: https://doi.org/10.1016/i.annals.2009.11.005

Mathews, S.J; Bonn, M.A; Snepenger, D. (2009) Atmospherics and consumers symbolic interpretations of hedonic services. International Journal of Culture, Tourism and Hospitality Research, 3(3), p. 193-210. DOI: https://doi.org/10.1108/17506180910980519

Mcintosh, A. J.; Prentice, R. C. (1999) Consuming Cultural Heritage. Annals of Tourism Research, 26(3), p. 589-612. DOI: https://doi.org/10.1016/S0160-7383(99)00010-9

Melucci, A. (2005) Busca da qualidade, ação social e cultura: por uma sociologia reflexiva. In: Melucci, A. Por uma sociologia reflexiva: pesquisa qualitativa e cultura. Petrópolis: Vozes.

Merriam, S.B. (1998) Qualitative research and case study application in education. São Francisco: Jossey-Bass.

Mkono, M. (2012) Authenticity Does Matter. Annals of Tourism Research, 39(1), p. 480483.DOi:

https://doi.org/10.1016/j.annals.2011.06.004

(2016) The reflexive tourist. Annals of Tourism Research, 57, p. 206-219. DOI: https://doi.org/10.1016/i.annals.2016.01.004

Moilanen, T.; Rainisto, S. (2009) How to Brand Nation, Cities and Destinations: a planning book for place branding. New York: Palgrave Macmillan. DOI: https://doi.org/10.1057/9780230584594

Morin, E. (2010) Introdução ao pensamento complexo. 4 ed. Porto Alegre: Sulina.

(2013) Ciência com consciência. 15. ed. Rio de Janeiro: Bertrand Brasil.

Moscardo, G. Pearce, P. (1986) Authenticity As a 
Travel Motive. Annals of Tourism Research, 13, p. 467-479. DOI: https://doi.org/10.1016/01607383(86)90031-9

Mossberg, L. Extraordinary Experiences through Storytelling. Scandinavian Journal of Hospitality and Tourism, 8(3), $2008 . \quad$ DOI: https://doi.org/10.1080/15022250802532443

Noy, C. (2004) This trip really changed me: Backpackers' narratives of self-change. Annals of Tourism Research, 31(1), p. 78-102.DOI: https://doi.org/10.1016/i.annals.2003.08.004

Nuryanti, W. (1996) Heritage and Postmodern. Annals of Tourism Research, 2, p. 249-260. DOI: https://doi.org/10.1016/0160-7383(95)00062-3

Patton, M. (2002) Qualitative research and evaluation methods. 3 ed. London: SAGE Publications.

Reisinger, Y.; Steiner, C. J. (2006) Reconceptualizing object authenticity. Annals of Tourism Research, 33(1), p. 65-86. DOI: https://doi.org/10.1016/j.annals.2005.04.003

Richards, G. (1996) Production and consumption of Eueopean cultural tourism. Annals of Tourism Research, 23(2), p. 261-283. DOI: https://doi.org/10.1016/0160-7383(95)00063-1

. (2003) Turismo creativo: uma nueva strategia? In Ortega, E. (ed) Investigación y estrategias turísticas. Madrid: Thomson, p. 107122.

. (2011) Criativity and Tourism: the state of the art. Annals of Tourism Research, 38(4), p. 1225-1253.

DOI:

https://doi.org/10.1016/j.annals.2011.07.008

Richards, G.; Wilson, J. (2006) Developing creativity in tourist experiences: a solution to the serial reproduction of culture? Tourism Management, 27, p. 1408-1413. DOI: https://doi.org/10.1016/i.tourman.2005.06.002

Rickly-Boyd, J. M. (2012) Authenticity \& aura: A benjaminian approach to tourism. Annals of Tourism Research, 39(1), p. 269-289. DOI: https://doi.org/10.1016/j.annals.2011.05.003

Rickly-Boyd, J. M. (2013) Alienation: Authenticity's Forgotten Cousin. Annals of Tourism Research, 40(1), p. 412-415. DOI: https://doi.org/10.1016/i.annals.2012.10.001

Robinson, R. N. S.; Clifford, C. (2012) Authenticity and festival foodservice experiences. Annals of Tourism Research, 39(2), p. 571-600. DOI: https://doi.org/10.1016/i.annals.2011.06.007

Salazar, N. B. (2005) Tourism and glocalization: "Local" tour guiding. Annals of Tourism Research, 32(3), p. 628-646. DOI: https://doi.org/10.1016/i.annals.2004.10.012

Scantlebury, M. M. G. (2011) Values in tourism: An itinerary to tourism ethics. Annals of Tourism Research, 38(2), p. 725-727. DOI: https://doi.org/10.1016/i.annals.2011.01.011

Schmitt, B.H. (1999) Experiential marketing: How to get customers to sense, feel, think, act, and relate to your company and brands. New York: NY: Free Press.

Nobel, 2000.

Marketing experimental. São Paulo:

Sedmak, G.; Mihali, T. (2008) Authenticity in mature seaside resorts. Annals of Tourism Research, 35(4), p. 1007-1031. DOI: https://doi.org/10.1016/i.annals.2008.07.004

Shepherd, R. J. (2015) Why Heidegger did not travel: existential angst, authenticity, and tourist experiences. Annals of Tourism Research, 52, p. 60-71.

DOI:

https://doi.org/10.1016/j.annals.2015.02.018

Skard, S.; Nysveen, H.; Pedersen, P. E. (2011) Brand and Customer Experience. In Service Organizations: Literature Review and Brand Experience Construct Validation. Institute For Research In Economics And Business Administration. SNF Working Paper No. 9.

Silver, I. (1993) Marketing Authenticity in Third World Countries. Annals of Tourism Research, 20, p. 302-318. DOI: https://doi.org/10.1016/0160- 


\section{$\underline{7383(93) 90057-A}$}

Smith, M.; Robinson, M. (2006) Cultural tourism in a changing world. Clevedon: Channel View Publications.

Stake, R. E. (1995) The Art of Case Study Research. London: SAGE Publications.

Steiner, C. J.; Reisinger, Y. (2006) Understanding existential authenticity. Annals of Tourism Research, 33(2), p. 299-318. DOI: https://doi.org/10.1016/i.annals.2005.08.002

Steiner, C.; Reisinger, Y. (2006) Reply to Belhassen and Caton. Annals of Tourism Research, 33(3), p. 853-856. DOI: https://doi.org/10.1016/i.annals.2006.03.001

Stovel, H. (2007) Effective Use of Authenticity and Integrity as World Heritage Qualifiying Conditions. City \& Time, 2(3), p. 21-36.

Sydney, S. (1996) Tourism, Heritage and Authenticity : State- Assisted Cultural Commodification in. PerspectivasUrbanas / Urban perspectives, p. 1-10.

Taylor, J. P. (2001) Authenticity and sincerity in tourism. Annals of Tourism Research, 28(1), p. 726. DOI: $\quad$ https://doi.org/10.1016/S01607383(00)00004-9

Thompson, E. (2007) Cognitive science and human experience. In E. Thompson. Mind in life: biology phenomenology, and sciences of mind. Cambridge: Harvard University Press.

Tribe, J.; Xiao, H. (2011) Developments in tourism social science. Annals of Tourism Research, 38(1), p. 7-26. DOI:

https://doi.org/10.1016/j.annals.2010.11.012

Tung, V. W. S.; Ritchie, J. R. B. (2011) Exploring the essence of memorable tourism experiences. Annals of Tourism Research, 38(4), p. 1367-1386. DOI: https://doi.org/10.1016/j.annals.2011.03.009

Uriely, N. (2005) The tourist experience. Conceptual developments. Annals of Tourism Research, 32(1), p. 199-216. DOI: https://doi.org/10.1016/j.annals.2004.07.008

Urry, J. (2003) The Sociology of Tourism. IN: Cooper, C. Classic Reviews in Tourism (cap 2). Clevedom: Channel View Publications.

Urry, J. (2002) The tourist gaze. Second edition. London: Sage.

Vasconcellos, M.J. (2013) Pensamento Sistêmico: o novo paradigma da ciência. 10 ed. Campinas: Papirus.

Verhoef, P. C.; Lemon, K. N.; Parasuraman, A; Roggeveen, A.; Tsiros, M.; Schlesinger, L. A. (2009) Customer Experience Creation: determinants, dynamics and management strategies. Journal of Retailing. 85, p. 31-41. DOI: https://doi.org/10.1016/j.jretai.2008.11.001

Waitt, G. (2000) Consuming heritage. Annals of Tourism Research, 27(4), p. 835-862. DOI: https://doi.org/10.1016/S0160-7383(99)00115-2

Waller, J.; Lea, E. G. S. (1999) Seeking the real spain?Authenticity in Motivation. Annals of Tourism Research, 26(1), p. 110-129. DOI: https://doi.org/10.1016/S0160-7383(98)00058-9

Wang, N. (1999) Rethinking authenticity in tourism experience. Annals of Tourism Research, 26(2), p. 349-370. DOI: https://doi.org/10.1016/\$0160-7383(98)00103-0

Wang, Y. (2007) Customized authenticity begins at home. Annals of Tourism Research, 34(3), p. 789-804.

DOI:

https://doi.org/10.1016/i.annals.2007.03.008

Weaver, A. (2005) The Mcdonaldization thesis and cruise tourism. Annals of Tourism Research, 32(2), p. 346-366. DOI: https://doi.org/10.1016/j.annals.2004.07.005

Weightman, B. A. (1987) Third World Tour Landscapes. Annals of Tourism Research, 14, p. 227-239. DOI: https://doi.org/10.1016/01607383(87)90086-7

Xiang, H.; Zhang, L. (2012) Key concepts in tourist studies. Annals of Tourism Research, 39(3), p. 
1735-1736.

https://doi.org/10.1016/j.annals.2012.05.012

Xin, S.; Tribes, J.; Chambers, D. (2013) Conceptual Research in Tourism. Annals of Tourism Research, 41, p. 66-88. DOI: https://doi.org/10.1016/i.annals.2012.12.003

Xue, L.; Manuel-Navarrete, D.; Buzinde, C. N. (2014) Theorizing the concept of alienation in tourism studies. Annals of Tourism Research, 44(1), p. 186-199. DOI:

https://doi.org/10.1016/i.annals.2013.10.001

Zomerdijk, L. G.; Voss, C. A. (2010) Service design for experience-centric services. Journal of Service Research, 13, p. 67-82. DOI: https://doi.org/10.1177/1094670509351960

Zhou, Q. et al. (2015) A structural model of host authenticity. Annals of Tourism Research, 55, p. 28-45.

DOI:

https://doi.org/10.1016/j.annals.2015.08.003

Zhu, Y. (2012) Performing heritage: Rethinking authenticity in tourism. Annals of Tourism Research, 39(3), p. 1495-1513. DOI: https://doi.org/10.1016/i.annals.2012.04.003
Informações dos autores

\section{Mariana Bueno de Andrade Matos}

Doutora em Administração pelo Programa de Pós-Graduação em Administração da UFPE (PROPAD/UFPE); Professora Adjunta do Departamento de Turismo e Hotelaria da Universidade Federal da Paraíba (DTH/UFPB).

E-mail: mbuenodeandrade@gmail.com ORCID: https://orcid.org/0000-0002-8723-3258

\section{Maria de Lourdes de Azevedo Barbosa}

Doutora em Administração pelo Programa de Pós-Graduação em Administração da Universidade Federal de Pernambuco (PROPAD/UFPE); Professora do Departamento de Hotelaria e Turismo da Universidade Federal de Pernambuco (DHT/UFPE); Coordenadora do Programa de Pós-Graduação em Hotelaria e Turismo da Universidade Federal de Pernambuco (PPHTUR/UFPE); Professora do Programa de Pós-Graduação em Administração da UFPE (PROPAD).

E-mail: lourdesbarbosa@gmail.com

ORCID: https://orcid.org/0000-0002-1790-380X 